\title{
Inhaltsverzeichnifs des fünften Bandes, nach den Gegenständen.
}

\section{Reine Mathematik.}

Nr. der
Ablandlung
1. An a $1 \dot{y} \mathrm{~s}$ i s.

Beft Seite

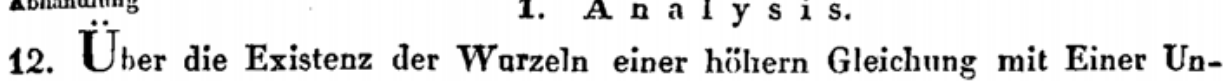
bekannten. Vom Hrn. Adam Burg, Professor der höhern Mathematik am

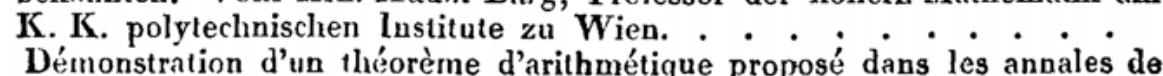

13. Démonstration d'un théorème d'arithmétique proposé dans les annales de mathémaliques de Mr. Gergonne, tom. XIX. p. 256. Par Mr. J. $A$. Grunert, prof. des math. a Brandebourg. $\dot{\text { Mémoire sur la convergence de la série du binome; pour faire suite à }}$

14. Mémoire sur la convergence de la série du binome; pour faire suite à
la démonstration du théorène du binốme, đonnée tome liI. de ce journal,

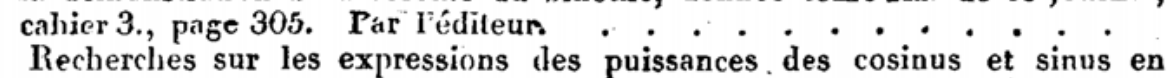
cosinus et sinus des arcs multiples, et sur les expressions réciprogñes. Par l'éditeur. . . . . . . . . . . . . . . . . . . . . . II. 197

21. Deux théorèmes sur les nombres.

23. Über Interpolation. Von Herrn Th. Clausen zu München. . . . . III. 305

27. De approximata seriei, juxta data functionis derivata dispositae, summatione. Auct. Dr, C J. D. Hill, Holm. . . . . . . . . . IV. 319

28. Mathematische Bruchstücke aus Herrn N. H. A bel's Briefen. . . IV. 336

29. Exercitatio algebraica circa discerptionem singularem fractionum, quae plures variabiles involvunt. Auct. C. G. J. Jacobi, prof. math. ord. Regiom. IV. 344

30. Über die Relationen der Functionen welche der Gleichung $F_{1} y \cdot \varphi_{1} x+F_{2} \gamma_{2} x \ldots+F_{n} y \cdot \varphi_{n} x=F_{1} x \cdot \varphi_{1} y+F_{2} x \cdot \varphi_{2} y \ldots+F_{n} x \cdot \varphi_{n} y$ genugthun. Von Herrn L. J. Magnus zu Berlin. . . . . . . . IV. 365

32. Über die Summs der Reine

$$
1+\frac{1}{2^{2}}+\frac{1}{3^{2}}+\frac{1}{3^{2}} \ldots \text { and } 1+\frac{1}{7^{2}}+\frac{1}{13^{2}}+\frac{1}{19^{2}} \ldots
$$

Von Herrn Th. Clausen zu München. . . . . . . . . . . IV. 380

34. Théorème sur les nombres. . . . . . . . • . . . . . . . IV. 386

35. Sur un principe génúral dans la théorie des séries. Mar Mr. de Schmidten, prof. des mathém. à Copenhague. . . . . . . . . . . IV. 388

37. Combinatorisch - analytische Abhandlung, enthaltend den Beweis der vier Summationsformeln Baud 3. Heft 2. S. 207. d. Journals. Von Herrn Pr. Gudermann zu Cleve.

1. Uber ein neues Coordinatensystem. Vom Herrn Professor Plücker za

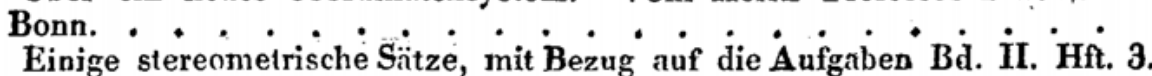
S. 292. No. 66. Vom Herrn Prof. Dr. Grunert zu Brandenburg. . .

5. Barycentrische Lösung der Aufgabe des Herrn $T h$. Cla usen in des IV. Bandes 4. Hefte, Seite 391. u. s. w. Vom Herrn Professor Möbius zu Leipzig.

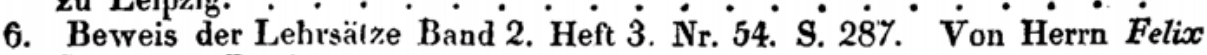

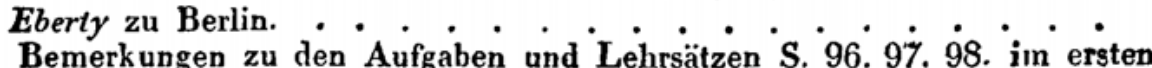

10. Bemerkungen zu den Aufgaben und Lehrsätzen S. 96. 9\%. 98. im ers
Heft zweiten Bandes dieses Journals. Voun Herrn O. G. D. Avbert Cloristiania in Norweren 
Nr. der

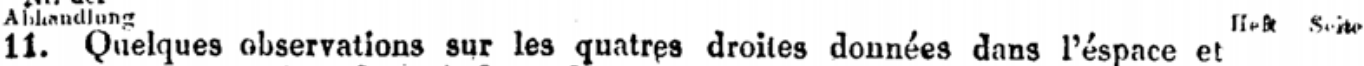
et non comprises deux â deux dans un même plan. Par Mr. Garbinsky', prof. à l'univ. et directeur de l'école polyt. à Varsovie.

18. Anwendung der elliptischen Transcendenten auf die sphärischen Polygone, welche zugleich einem kleinen Kreise der Kugel eingeschrieben und einem andern umgeschriehen sind. Von Hrn. Stud. Richclot zu Königsberg in Pr. III. 230

19. Über ein neues Princip der Geometrie und den Gebrauch allgemeiner Symbole und unbestimmter Coëfficienten. Von Hrn. Prof. Plïcker zu Bonn. III. 268

22. Über die Curven des kürzesten l'erimeters auf krummen Flächen. In Folge der Aufgabe 6. Band 3. Heft 1. S. 99. Von Herrn Dr. Ferd. Minding zu Berlin. • • • • • • • • • • • • • • • • • •

25. Beweis eines Lehrsatzes vom Fünfecke. In Folge deri Aufstellung desselben S. 396., 4. Band 4. Heft dieses Journals. Von Hrn. G........ III. 316,

36. Auflösung einiger Aufgaben der analytischen Geometrie vermittelst des barycentrischen Calculs. Von dem. Herrn Ober-Lehrer F. Minding zu Berlin.

3. M e c h a n i k.

4. Recherches sur la figure et le mouvement d'une bulle d'air, dans un liquide de densité constante; question proposée par l'Academie Royale de Bruxelles pour le concours de 1828. Par Mr. Theremin, Capitaine du génie des voies de communications à Ircoutsk en Sibérie. . . . .

9. Mémoire sur le mouvement d'un corps rigide, soutenu par un plan fixe. Par Mr. A. A. Cournot, Dr. ès sciences à Paris. - . - . - .

17. Du mouvement d'un corps sur un plan fixe, quand on a égard a la résistance du frottement, et qu'on ne suppose qu'un seul point de contact. Par Mr. A. A. Cournot, Dr. ès sciences à Paris. (Suile du mémoire

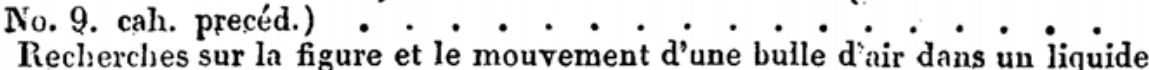

31. Recherclses sur la figure et le mouvement d'une bulle d'air dans un liquide
de densité constante. Par Mr. Theremin, capitaine du gérie des voíes de communications à Ircoutsk en Sibérie. (Suite du mémoire No. 4. tom. V. cah. 1.) • • . • • • • • , • • • • • • • • • • • • IV. 374

33. Über die Bestimmung der Lage der Haupt-Undrelıungs-Axen eines Körpers, Von Herrn Th. Clausen zu München. . . . . . . . IV. 383

\section{Angewandte Mathematik.}

3. Allgemeine und vollständige Berechnung aller beim Gleichgewichte mit Rïcksicht auf Zapfenreibung vorkommenden Bestimmungsstücke. Von dem Herrn Dr. G. S. Ohm zu Berlin. . . . . . . . . . . I. 51

8. Kurze Darstellung der Haupt-Eigenschafien eines Systems von Linsengläsern. Vom Herrn Prof. A. F. Möbius zu Leipzig. . . • • . II. 113

20. Solution d'une question relative à la théorie mathématique de la chaleur. Par Mr. Lejeune-Dirichlet, prof. de mathém. . . . . . . . . III. 287

24. Über Centrifugal - Pendel - Uhren. Von Hrn. Th. Clausen zu München. III. 314 'Aufgaben und Lehrsätze.

7. Aufgaben und Lehrsätze, erstere aufzulösen, letztere zu beweisen; nebst anderen einzelnen Bemerkungen. • . . . . . . . . . . . . I. 110

16. Aufgabe. . . . . . . . . . . . • . . . . . . . . . II. 222

26. Aufgaben und Lehrsätze, erstere aufzulösen, letztere zu berveisen. - III. 317

38. Einige Nachrichten von Büchern. . . . . . . . . . . . . IV. 414 